\title{
Hochtemperatur-Ultraschallwandler zur kontinuierlichen Strukturüberwachung von Dampfleitungen
}

\author{
Dr. Bernhard Brunner, Fraunhofer Institut Silicatforschung, Würzburg
}

\section{Zusammenfassung:}

Zur Strukturüberwachung an heißen Rohrleitungen wurden spezielle Hochtemperatur-Ultraschallwandler entwickelt, die kontinuierlich den Zustand der Stahlrohrleitung bezüglich Risse bzw. deren Entstehung und großflächiger Oberflächenkorrosion analysiert. Da konventionelle auf Blei-Zirkonat-Titanat (PZT) basierende Ultraschallwandler wegen ihrer niedrigen Curietemperatur nicht für den Einsatz bei Rohroberflächentemperaturen von $500-700^{\circ} \mathrm{C}$ geeignet sind, wurden Wandler auf Basis von LangasitEinkristallen entwickelt, die mit speziellen Glasloten passiviert und mechanisch auf der Stahlrohroberfläche fixiert werden. Erste Labortests ergaben Ultraschallüberwachungsstrecken von einigen Metern.

\section{Motivation:}

Schäden an funktions- und sicherheitsrelevanten Strukturbauteilen verursachen in verschiedenen Industriebereichen hohe Kosten. Um Defekte rechtzeitig zu entdecken und unnötige Stillstandzeiten oder gar Unfälle zu vermeiden, ist in der Regel ein hoher Wartungsaufwand erforderlich. Mit Hilfe von innovativen Überwachungskonzepten können Betriebszeiten technischer Anlagen verlängert, damit Wartungskosten erheblich gesenkt und somit die Wirtschaftlichkeit technischer Anlagen nachhaltig gesteigert werden. Bei einem solchen Condition Monitoring System wird die Funktion der Bauteile periodisch oder kontinuierlich mit Hilfe von Sensoren vor Ort überwacht. Statt die Lebensdauer sicherheitsrelevanter Bauteile wie bisher auf Basis starrer Wartungsregimes aufwändig zu analysieren, ermöglichen neuartige Sensorkonzepte die Umstellung auf zustandsabhängige Wartungsmaßnahmen.

Gängige Überwachungskonzepte, die auf glasfaseroptischen Messungen, Beschleunigungs- oder Dehnungsmessungen beruhen, detektieren nur größere Fehler und können diese auch nicht genau orten. Ultraschall hingegen eignet sich besonders gut, um feine $\left(\sim \mathrm{mm}^{2}\right)$ Strukturschäden in Glas- oder Kohlefaserkompositen, aber auch in Stahlbauteilen zu entdecken. Für den Einsatz in einer Hochtemperaturumgebung sind herkömmliche auf Blei-Zirkonat-Titanat (PZT) basierte Sensoren aufgrund ihrer zu geringen CurieTemperatur nicht geeignet. Eigens für diese Anwendung am Fraunhofer ISC entwickelte HochtemperaturUltraschallwandler basieren auf Einkristallmaterialien (z.B. Langasit), die zur Passivierung - und auch zur späteren Befestigung der Wandler am Einsatzort - in Spezialglaslot eingebettet sind.

Damit wird es erstmals möglich, bis $700^{\circ} \mathrm{C}$ heiße Rohrleitungen oder Behälter von Chemieanlagen und Kraftwerken kontinuierlich während des Betriebs zu überwachen und die gegenwärtigen Prozess- und Überwachungsparameter Druck und Durchfluss zu ergänzen. Leckagen in Rohrleitungen werden also nicht erst nach ihrem Auftreten entdeckt, sondern können vorab schon in der Entstehungsphase lokalisiert werden. Dadurch entstehende Betriebsausfälle können somit weitgehend vermieden werden.

\section{Wandleraufbau:}

Die Hochtemperatur-Ultraschallwandler bestehen aus einem Langasit-Einkristall $\left(\mathrm{La}_{3} \mathrm{Ga}_{5} \mathrm{SiO}_{14}\right.$, y-cut $)$ mit einer Fläche von 15 x $15 \mathrm{~mm}^{2}$ und einer Dicke von ca. $0.5 \mathrm{~mm}$, der auf beiden Seiten mit gesputterten Platinoder Goldelektroden beschichtet ist. Die elektrische Kontaktierung erfolgt über geschirmte Platindrähte mit einer Länge bis zu einem Meter. Sowohl zur Passivierung des gesamten Wandleraufbaus als auch zur elektrischen Isolierung gegenüber dem Dampfleitungsrohr werden spezielle Glaslote mit unterschiedlichen thermischen Ausdehnungskoeffizienten und Glasübergangstemperaturen eingesetzt. Dadurch wird es auch möglich, die großen Unterschiede in den thermischen Ausdehnungskoeffizienten des Langasits gegenüber 
dem Stahlrohr anzupassen und Dämpfungskörper zu gestalten. Bild 1 zeigt schematisch den Wandleraufbau auf einem Stahlrohr.

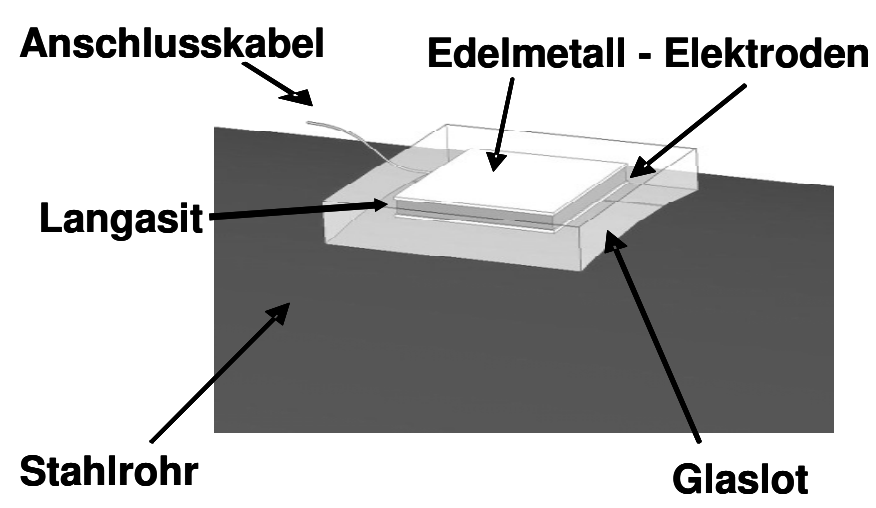

Bild 1: Aufbau des Hochtemperatur-Ultraschallwandlers

Die Wandler weisen somit eine Gesamtdicke von etwa drei Millimetern und eine Fläche von nur wenigen Quadratzentimetern auf (Bild 2). Dadurch ist es möglich, diese auch auf gekrümmten Rohroberflächen mit Hilfe von Glasloten, die speziell an die Betriebstemperatur und an das thermische Ausdehnungsverhalten des Rohrmaterials angepasst sind, zu fixieren.

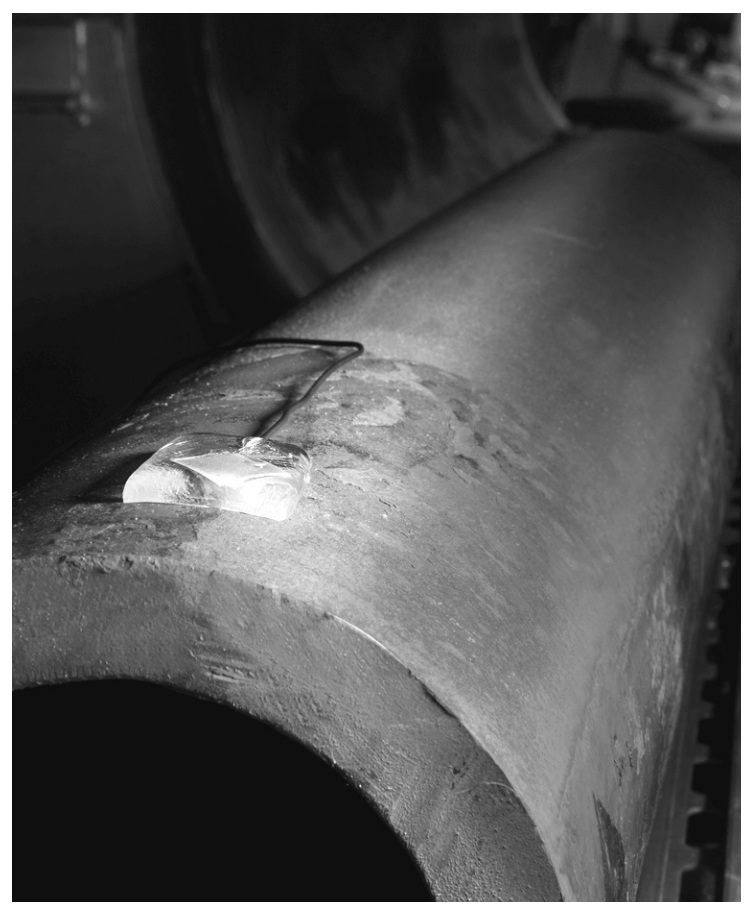

Bild 2: Applikation eines Hochtemperatur-Ultraschallwandlers auf einem Dampfleitungsrohrstück

\section{Messprinzip:}

Mithilfe der Steuerungselektronik werden die piezoelektrischen Wandler zu Ultraschallschwingungen im Frequenzbereich von $50-500 \mathrm{kHz}$ angeregt, die sich in die Bauteiloberfläche übertragen. Das dabei entstehende Wellenmuster dieser Ultraschall-Lambwellen wird an ein Empfangsgerät übertragen. Risse oder 
großflächige Korrosion verändern das gleichmäßige Wellenfeld und lassen sich so leicht detektieren. Da die Wandler abwechselnd sowohl als Empfänger (Sensor) als auch als Ultraschallsender (Aktor) fungieren können, lassen sich an verschiedenen Stellen des Bauteils gezielt Wellenfronten erzeugen und selbst versteckte Winkel prüfen. Die von den Wandlern ausgesendeten und reflektierten Ultraschallimpulse werden über die Elektronik mit dem störungsfreien Soll-Zustand verglichen. Über die Laufzeitmessungen lassen sich Fehler auf wenige $\mathrm{cm}$ genau lokalisieren. Das Ultraschallfeld eines Wandlers reicht etwa drei Meter weit, so dass bereits wenige davon genügen, um ein lückenloses Sensornetzwerk an den besonders gefährdeten Stellen von heißen Rohrleitungen zu etablieren.

\section{Ergebnisse:}

Bild 3 zeigt das Transfersignal zwischen einem Langasit-Hochtemperaturwandler und einem PZT- Referenzwandler bei einem Abstand von drei Metern auf einem Dampfleitungsrohr. Die Messungen wurden im Labor bei Raumtemperatur bei einer Frequenz von $500 \mathrm{kHz}$ und einer Sendeamplitude von $200 \mathrm{~V}_{\mathrm{pp}}$ durchgeführt.

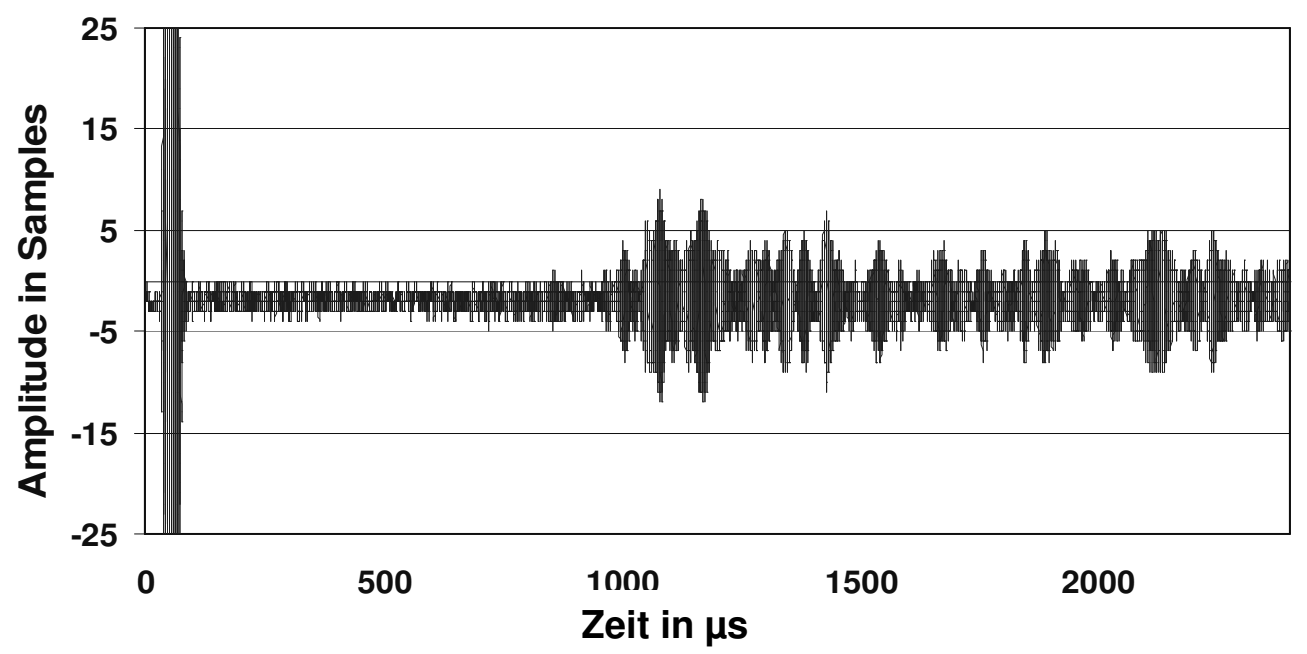

Bild 3: Empfangssignal eines Langasit-Hochtemperaturwandlers bei einem Abstand von drei Metern zum Sender

Deutlich erkennbar sind empfangenen Longitudinal- und Transversalwellen nach einer Laufzeit von ca. 1100 $\mu$ s. Die zeitlich etwas später eintreffenden schwächeren Schallereignisse stammen von Mehrfachreflexionen der Wellenpakete an den Rohrenden des insgesamt etwa vier Meter langen Rohrleitungsstückes im Labor.

Eine Optimierung des Wandleraufbaus und Verbesserungen in der elektrischen Ansteuerung sollen die Signalstärken erhöhen und erlauben dann Feldtests an längeren etwa $500^{\circ} \mathrm{C}$ heißen Dampfleitungen in einem Kraftwerk. 